Médecine \& Maladies infectieuses (2016) vol 46 Nº spécial EMERG

\title{
Établissement d’une présence endémique de la Bilharziose en Corse
}

Berry (1), J. Fillaux (1), G. Martin-blondel (1), J. Boissier (2), X. Iriart (1), B. Marchou (1), J. Magnaval (1), P. Delobel (1)

(1) CHU Purpan, Toulouse,

(2) Université de Perpignan Via Domitia.

\section{Introduction}

Une épidémie de bilharziose urinaire est survenue au cours de l'été 2013 en Corse, dans la région de Porto-Vecchio (rivière Cavu). Par la suite, une vaste enquête épidémiologique avait été effectuée pour dépister et traiter les porteurs de Schistosomes. Aucun cas n'avait été rapporté depuis. Nous rapportons trois nouveaux cas acquis au cours de l'été 2015 qui démontrent l'établissement d'une présence endémique de la Bilharziose urinaire en Corse.

\section{Matériels et méthodes}

Nous rapportons trois cas de primo-infection à Schistosomia haematobium dans leurs aspects cliniques, biologiques, et épidémiologiques. La séroconversion a été objectivée par ELISA, hémagglutination indirecte, et Western-Blot. Les données épidémiologiques ont été établies par questionnaire des sujets exposés et cartographie des lieux de baignade.

\section{Résultats}

L'infection aiguë est attestée par une séro-conversion pour Schistosomia haematobium, accompagnée parfois de signes cliniques et d'hyperéosinophilie. L'enquête épidémiologique a retrouvé pour les 3 cas des baignades dans la rivière Cavu en Corse au cours de l'été 2015, à l'exclusion de toute autre source potentielle de contamination. Le lien avec l'épidémie de 2013 est probable mais le mécanisme de persistance n'est pas établi. La réintroduction dans la rivière par l'urine de sujets non dépistés lors de la campagne de 2013 est l'hypothèse la plus probable.

\section{Conclusion}

Ces nouveaux cas de bilharziose urinaire suite à des baignades dans la rivère Cavu au cours de l'été 2015 démontrent l'établissement d'une présence endémique de la Bilharziose en Corse. Une diffusion dans d'autres rivières du Sud de l'Europe, où le mollusque hôte intermédiaire est présent, ne peut être exclue et nécessite une surveillance épidémiologique.

Aucun lien d'intérêt 